\title{
BMC Family Practice reviewer acknowledgment 2015
}

Elaine Zhang

\section{Contributing reviewers}

The editors of BMC Family Practice would like to thank all our reviewers who have contributed to the journal in Volume 16 (2015)

Arye Abeles

Oladele Vincent Adeniyi

South Africa

Girdhar Agarwal

India

Sarah Alderson

Kelli Allen

USA

Muhammad Alotaibi

Kuwait

Grove Amy

UK

Foteini Anastasiou

Greece

Angeliki Angelidi

Greece

Aniela Angelow

Germany

Eva Arvidsson

Sweden

Diane Ashiru-Oredope

UK

Felicity Astin

UK
Salla Atkins

Sweden

Kris Aubrey-Bassler

Canada

Luciana Ballini

Italy

Paola Ballotari

Italy

Michelle Banfield

Australia

Yael Bar Zeev

Israel

Marie Barais

France

Elizabeth Barley

UK

Karen Barnett

UK

Heather Barry

UK

Christopher Barton

Australia

Maria Basta

Greece

Per Bech

Denmark

\author{
Iain Beith \\ UK
}

Ann Berger

USA

Christophe Berkhout

France

Annette Bishop

UK

Lindsay Blank

UK

Jutta Bleidorn

Germany

\section{Jeanet Blom}

Netherlands

Eva Blozik

Germany

Femke Boehmer

Germany

Wienke Boerma

Netherlands

Fiona Boland

Ireland

Gunnar Bondevik

Norway

S Boodai

UK

Correspondence: elaine.zhang@biomedcentral.com

BioMed Central, Floor 6, 236 Gray's Inn Road, London WC1X 8HB, UK 
Helen P. Booth

UK

Marije Bosch

Australia

Marie Bradley

USA

Kate Brain

UK

Paula Brauer

Canada

Iain Brew

UK

Bianca Brijnath

Australia

Eileen Britt

New Zealand

B.D. Lidewij Broekhuizen

Netherlands

Lisa Brunton

UK

Alessandra Buja

Italy

Christopher Burton

UK

Joan Busfield

UK

Jo Byrne

UK

Paul Campbell

UK

Stephen Campbell

UK

Christine Carius

Germany

Melinda Carrington

Australia

Yasemin Çayır

Turkey

Venija Cerovecki

Croatia

Gülsen Ceyhun Peker

Turkey

Tom Chan

UK
Anna Cheshire

UK

Rie Chiba

Japan

Kyoung Im Cho

Korea, South

Fatma Cihan

Turkey

David J Clarke

UK

John Cleland

UK

Manuel J. Cobo

Spain

Naama Constantini

USA

Carol Coole

UK

Vincent Cooper

UK

Suzanne Cosh

Australia

Elizabeth Cottrell

UK

Peter Alan Coventry

UK

Alan Crockett

Australia

Jesse Crosson

USA

Sarah Cutrona

USA

Slawomir Czachowski

Poland

Carl D'Arcy

Canada

Eefje De Bont

Netherlands

Daan De Coster

UK

Corina De Jong

Netherlands

Jip De Jong

Netherlands
Jan De Maeseneer

Belgium

An De Sutter

Belgium

Marcelo Demarzo

Brazil

Catherine Demers

Canada

Sophie Derchain

Brazil

Tobias Deutsch

Germany

Fiona Doolan-Noble

New Zealand

Athanassios Douzenis

Greece

Frank Doyle

Ireland

Alex Dregan

UK

Sinead Duane

Ireland

Anthony D'Urzo

Canada

Anne G. Ekeland

Norway

Nouhad El-Haddad

Australia

Rose Ellis

Australia

Jon Emery

Australia

Romain Eschalier

France

Evangelos Evangelou

Greece

Karen Fairhurst

UK

Andrew Farmer

UK

Nighat Faruqi

Australia

Annunziata Faustini Italy 


\begin{tabular}{|c|c|c|}
\hline $\begin{array}{l}\text { Ahmad Fayaz-Bakhsh } \\
\text { Iran }\end{array}$ & $\begin{array}{l}\text { Paolo Giorgi Rossi } \\
\text { Italy }\end{array}$ & $\begin{array}{l}\text { Erik Hemmingsson } \\
\text { Sweden }\end{array}$ \\
\hline $\begin{array}{l}\text { Gregor Feldmeier } \\
\text { Germany }\end{array}$ & $\begin{array}{l}\text { Dianne Goeman } \\
\text { Australia }\end{array}$ & $\begin{array}{l}\text { Wolfgang Himmel } \\
\text { Germany }\end{array}$ \\
\hline $\begin{array}{l}\text { Julie Ferguson } \\
\text { UK }\end{array}$ & $\begin{array}{l}\text { Katja Goetz } \\
\text { Germany }\end{array}$ & $\begin{array}{l}\text { Samuel Ho } \\
\text { USA }\end{array}$ \\
\hline $\begin{array}{l}\text { Bart Ferket } \\
\text { USA }\end{array}$ & $\begin{array}{l}\text { Fatma Gökşin Cihan } \\
\text { Turkey }\end{array}$ & $\begin{array}{l}\text { Robert Hoffman } \\
\text { Israel }\end{array}$ \\
\hline $\begin{array}{l}\text { Rachael Finn } \\
\text { UK }\end{array}$ & $\begin{array}{l}\text { Debora Goldberg } \\
\text { USA }\end{array}$ & $\begin{array}{l}\text { Rhona Hogg } \\
\text { UK }\end{array}$ \\
\hline $\begin{array}{l}\text { Emily Fletcher } \\
\text { UK }\end{array}$ & $\begin{array}{l}\text { Trish Gorely } \\
\text { UK }\end{array}$ & $\begin{array}{l}\text { Knut Holtedahl } \\
\text { Norway }\end{array}$ \\
\hline $\begin{array}{l}\text { Michelle Foley } \\
\text { UK }\end{array}$ & $\begin{array}{l}\text { Martine Granek-Catarivas } \\
\text { Israel }\end{array}$ & $\begin{array}{l}\text { Guro Huby } \\
\text { Norway }\end{array}$ \\
\hline $\begin{array}{l}\text { Lynne Forrest } \\
\text { UK }\end{array}$ & $\begin{array}{l}\text { Aileen Grant } \\
\text { UK }\end{array}$ & $\begin{array}{l}\text { John Hughes } \\
\text { UK }\end{array}$ \\
\hline $\begin{array}{l}\text { Yvonne Forsell } \\
\text { Sweden }\end{array}$ & $\begin{array}{l}\text { Marcia Grant } \\
\text { USA }\end{array}$ & $\begin{array}{l}\text { Kate Hunt } \\
\text { UK }\end{array}$ \\
\hline $\begin{array}{l}\text { Nick Francis } \\
\text { UK }\end{array}$ & $\begin{array}{l}\text { Ben Gray } \\
\text { New Zealand }\end{array}$ & $\begin{array}{l}\text { Deirdre Hurley Osing } \\
\text { Ireland }\end{array}$ \\
\hline $\begin{array}{l}\text { Toby Freeman } \\
\text { Australia }\end{array}$ & $\begin{array}{l}\text { Eva Grunfeld } \\
\text { Canada }\end{array}$ & $\begin{array}{l}\text { Sylvia Hysong } \\
\text { USA }\end{array}$ \\
\hline $\begin{array}{l}\text { Thomas Frese } \\
\text { Germany }\end{array}$ & $\begin{array}{l}\text { Anna Gryko } \\
\text { Poland }\end{array}$ & $\begin{array}{l}\text { Eliza Iatraki } \\
\text { Greece }\end{array}$ \\
\hline $\begin{array}{l}\text { John Furler } \\
\text { Australia }\end{array}$ & $\begin{array}{l}\text { Jane Gunn } \\
\text { Australia }\end{array}$ & $\begin{array}{l}\text { Fumiaki Imamura } \\
\text { UK }\end{array}$ \\
\hline $\begin{array}{l}\text { Daniel Fuster } \\
\text { Spain }\end{array}$ & $\begin{array}{l}\text { Aimin Guo } \\
\text { China }\end{array}$ & $\begin{array}{l}\text { Baruch Itzhak } \\
\text { Israel }\end{array}$ \\
\hline $\begin{array}{l}\text { Ildiko Gagyor } \\
\text { Germany }\end{array}$ & $\begin{array}{l}\text { Anders Halling } \\
\text { Denmark }\end{array}$ & $\begin{array}{l}\text { Pupalan Iyngkaran } \\
\text { Australia }\end{array}$ \\
\hline $\begin{array}{l}\text { Maria Garcia-Gil } \\
\text { Spain }\end{array}$ & $\begin{array}{l}\text { Janet Hanley } \\
\text { UK }\end{array}$ & $\begin{array}{l}\text { Sally Jacobs } \\
\text { UK }\end{array}$ \\
\hline $\begin{array}{l}\text { Poonam Gardner-Sood } \\
\text { UK }\end{array}$ & $\begin{array}{l}\text { Shamil Haroon } \\
\text { UK }\end{array}$ & $\begin{array}{l}\text { Masahito Jimbo } \\
\text { USA }\end{array}$ \\
\hline $\begin{array}{l}\text { Natalie Gauld } \\
\text { New Zealand }\end{array}$ & $\begin{array}{l}\text { Mark Harris } \\
\text { Australia }\end{array}$ & $\begin{array}{l}\text { Hanna Kaduszkiewicz } \\
\text { Germany }\end{array}$ \\
\hline $\begin{array}{l}\text { Chris Gibbons } \\
\text { UK }\end{array}$ & $\begin{array}{l}\text { Jo Hart } \\
\text { UK }\end{array}$ & $\begin{array}{l}\text { Vera Kalitzkus } \\
\text { Germany }\end{array}$ \\
\hline $\begin{array}{l}\text { Christopher Gidlow } \\
\text { UK }\end{array}$ & $\begin{array}{l}\text { Johannes Hauswaldt } \\
\text { Germany }\end{array}$ & $\begin{array}{l}\text { Sebastian Kalwij } \\
\text { UK }\end{array}$ \\
\hline $\begin{array}{l}\text { Antonio Z Gimeno García } \\
\text { Spain }\end{array}$ & $\begin{array}{l}\text { Rebecca Hays } \\
\text { UK }\end{array}$ & $\begin{array}{l}\text { Khaled Karkabi } \\
\text { Israel }\end{array}$ \\
\hline $\begin{array}{l}\text { Carlo Bruno Giorda } \\
\text { Italy }\end{array}$ & $\begin{array}{l}\text { Christian Helfrich } \\
\text { USA }\end{array}$ & $\begin{array}{l}\text { Marise Kasteleyn } \\
\text { Netherlands }\end{array}$ \\
\hline
\end{tabular}


Paul Kelly
UK
Jakub Kenig
Poland
Anne Kennedy
UK
Natalie Kennie-Kaulbach
Canada
Cassandra Kenning
UK
Amanda Kenny
Australia
Myrna Keurhorst
Netherlands
Chris Keyworth
UK

Gholam Reza Kheirabadi Iran, Islamic Republic Of

Moira Kinnear
UK

Bruce Kirenga

Uganda

Spyridon Klinis

Greece

Louise Knight

UK

Sarah Knowles

UK

Lee Yew Kong

Malaysia

Kurt Kroenke

USA

Edward Krupat

USA

Donata Kurpas

Poland

Uwe Kurzke

Germany

Cindy Lam

Hong Kong

Maaike Langelaan

Netherlands

Susanne Langer

UK
Birgitta Langhammer

Norway

Michel Langlois

Belgium

Miranda Laurant

Netherlands

Rachel Laws

Australia

Patricia Lebensohn

USA

Marieke B Lemiengre

Belgium

Wei Shen Lim

UK

Alyson Littman

USA

Carl Lombard

South Africa

Daniel Lopez-Cevallos

USA

Fabiana Lorencatto

UK

Marta Losa Iglesias

Spain

Alexander Lustman

Israel

Richard Ma

UK

Annabelle Machin

UK

Parker Magin

Australia

Elizabeth Magnan

USA

Nicola Magnavita

Italy

Chris Maher

Australia

Helle Terkildsen Maindal

Denmark

Lilach Malatskey

Israel

Navjeet Mangat

UK
Adelais Markaki

Greece

Gabriella Marx

Germany

Edita Masanauskiene

Lithuania

Neal Maskrey

UK

Ioannis Mastoris

USA

Andre Matalon

Israel

Shin Matsuoka

Japan

Milena Maule

Italy

Mary Mccarron

Ireland

Serena Mccluskey

UK

Ron Mcdowell

Ireland

Sarah Mclachlan

UK

Kumara Mendis

Sri Lanka

Gabriele Messina Italy

Ilias Migdalis

Greece

Wendy Miller

USA

Mirella Minkman

Netherlands

Matthew D. Mitchell USA

Erin Moore

USA

Andrew Morden

UK

Frank Moriarty

Ireland

Rebecca Morris

UK 
Melanie Morris

UK

Luke Mounce

UK

Bozena Mroczek

Poland

Jean Muris

Netherlands

Edel Murphy

Ireland

Lucio Naccarella

Australia

Cristiana Nascimento-Carvalho

Brazil

Pauline Nelson

UK

David Neubauer

USA

Eva Neufeld

Canada

Evangelia Ntzani

Greece

Peter Nugus

Canada

Austin O Carroll

Ireland

Brendan O Shea

Ireland

Maeve O'Beirne

Canada

Allison Ober

USA

Amy O'Donnell

UK

Nelly Oelke

Canada

Jodie Oliver-Baxter

Australia

Braden O'Neill

Canada

Juan Fco Orueta

Spain

Brendan O'Shea

Ireland
Alice Owen

Australia

Alis Özçakır

Turkey

Mehmet Özen

Turkey

Zlata Ozvacic Adzic

Croatia

Leonidas Palaiodimos

Greece

Maria Panagioti

UK

Kosmas Paraskevas

Greece

Deborah Parra-Medina

USA

Zoe Paskins

UK

Winifred Paulis

Netherlands

Christina Pearce

UK

Emily Peckham

UK

Marta Pelayo

Spain

Michael Pentzek

Germany

Giulio Perugi

Italy

Davorina Petek

Slovenia

Marija Petek ¿Ter

Slovenia

Ferdinando Petrazzuoli

Sweden

Ferdinando Petrazzuoli

Italy

Mila Petrova

UK

Carlo Piccinni

Italy

Tamar Pincus

UK
Hilary Pinnock

UK

Maria Carmen Portillo

Spain

Rebekah Pratt

USA

Ivanka Prichard

Australia

Emmanuel Prokopakis

Greece

Sue Pullon

New Zealand

Martine Puts

Canada

Jennifer Quint

UK

Pietro Ragni

Italy

Vasiliki Rahimzadeh

Canada

Anil Rane

India

Elena Ratschen

UK

Robin Ray

Australia

Genevieve Rayner

Australia

Sabi Redwood

UK

Carole Reeve

Australia

Joanne Reeve

UK

David Reeves

UK

Darlene Reid

Canada

Zeljko Reiner

Croatia

Cristina Renzi

UK

Suzanne Richards

UK 
Eckhard Rickels

Germany

Herbert Riechelmann

Austria

Diane Roberts

UK

Charo Rodriguez

Canada

Anne Elizabeth Rogers

UK

Aldo Rosano

Italy

Marianne Rosendal

Denmark

Danica Rotar-Pavlic

Slovenia

Eugene Ruzagira

Uganda

Caroline Sanders

UK

Sally Sargeant

Australia

Kathleen Sarmiento

USA

Willemijn Schäfer

Netherlands

Jeffrey Scherrer

USA

Kathrin Schlößler

Germany

Guido Schmiemann

Germany

Antoinette Schoenthaler

USA

Antonis Schoinas

Greece

Jane Scott

Australia

Polona Selic

Slovenia

Turan Set

Turkey

Joshua Shadd

Canada
Michal Shani

Israel

James Sheppard

UK

Damin Si

Australia

Jonathan Silverman

UK

Rosemary Simmonds

UK

Panagiotis Simos

Greece

Aloysius Niroshan Siriwardena

UK

Susan Slade

Australia

Sarah Slight

UK

Nicola Small

UK

Susan Smith

Ireland

Sarah Smithson

UK

Emmanouil Smyrnakis

Greece

Ratna Sohanpal

UK

Robert Sokol

USA

Andreas Sonnichsen

Germany

Jaime Sousa

Portugal

Sheryl Spithoff

Canada

Melinda Stanners

Australia

Ellen Stein

USA

Aleksander Stepanović

Slovenia

Fiona Stevenson

UK
Scott Stewart

USA

Nigel Stocks

Australia

Matej Strnad

Slovenia

Andre Strydom

UK

Joachim Sturmberg

Australia

Frank Sullivan

Canada

Rachael Summers

UK

Patricia Sunaert

Belgium

Frederick Sundram

New Zealand

Elisabeth Svensson

Sweden

Emmanouil Symvoulakis

Greece

Adam G Tabak

UK

Mary R Talen

USA

Ryan Tandjung

Switzerland

Athina Tatsioni

Greece

Essa Tawfiq

New Zealand

Meredith Temple-Smith

Australia

Emma Thomas-Jones

UK

Hans Thulesius

Sweden

Petter Tinghog

Sweden

Els Tobback

Belgium

Gail Todd

South Africa 


\begin{tabular}{|c|c|c|}
\hline $\begin{array}{l}\text { Pinar Topsever } \\
\text { Turkey }\end{array}$ & $\begin{array}{l}\text { Jane Vennik } \\
\text { UK }\end{array}$ & $\begin{array}{l}\text { Connie Wiskin } \\
\text { UK }\end{array}$ \\
\hline $\begin{array}{l}\text { Helen Triantafyllidi } \\
\text { Greece }\end{array}$ & $\begin{array}{l}\text { Jan Verbakel } \\
\text { Belgium }\end{array}$ & $\begin{array}{l}\text { Maria Wolters } \\
\text { UK }\end{array}$ \\
\hline $\begin{array}{l}\text { Katrina Tsang } \\
\text { Hong Kong }\end{array}$ & $\begin{array}{l}\text { Peter Verhaak } \\
\text { Netherlands }\end{array}$ & $\begin{array}{l}\text { Carmen Wong } \\
\text { Hong Kong }\end{array}$ \\
\hline $\begin{array}{l}\text { Hedda Tschudi-Madsen } \\
\text { Norway }\end{array}$ & $\begin{array}{l}\text { Theo Verheij } \\
\text { Netherlands }\end{array}$ & $\begin{array}{l}\text { Christopher Wong } \\
\text { UK }\end{array}$ \\
\hline $\begin{array}{l}\text { Raymond Tweheyo } \\
\text { UK }\end{array}$ & $\begin{array}{l}\text { Areti Angeliki Veroniki } \\
\text { Canada }\end{array}$ & $\begin{array}{l}\text { Rachael Wood } \\
\text { UK }\end{array}$ \\
\hline $\begin{array}{l}\text { Fiona Ulph } \\
\text { UK }\end{array}$ & $\begin{array}{l}\text { Evelyn Vingilis } \\
\text { Canada }\end{array}$ & $\begin{array}{l}\text { Fiona Wood } \\
\text { UK }\end{array}$ \\
\hline $\begin{array}{l}\text { Jonathan Underhill } \\
\text { UK }\end{array}$ & $\begin{array}{l}\text { Shlomo Vinker } \\
\text { Israel }\end{array}$ & $\begin{array}{l}\text { James Woodall } \\
\text { UK }\end{array}$ \\
\hline $\begin{array}{l}\text { Christine Urquhart } \\
\text { UK }\end{array}$ & $\begin{array}{l}\text { Jaco Voorham } \\
\text { Netherlands }\end{array}$ & $\begin{array}{l}\text { Elizabeth Worsley } \\
\text { UK }\end{array}$ \\
\hline $\begin{array}{l}\text { Michael Van Den Berg } \\
\text { Netherlands }\end{array}$ & $\begin{array}{l}\text { Adrian Wagg } \\
\text { Canada }\end{array}$ & $\begin{array}{l}\text { Nat Wright } \\
\text { UK }\end{array}$ \\
\hline $\begin{array}{l}\text { Ann Van Den Bruel } \\
\text { UK }\end{array}$ & $\begin{array}{l}\text { Brian Walitt } \\
\text { USA }\end{array}$ & $\begin{array}{l}\text { Tina Wulff } \\
\text { Germany }\end{array}$ \\
\hline $\begin{array}{l}\text { Pieter Van Den Hombergh } \\
\text { Netherlands }\end{array}$ & $\begin{array}{l}\text { Anne Walsh } \\
\text { Australia }\end{array}$ & $\begin{array}{l}\text { Gwenllian Wynne-Jones } \\
\text { UK }\end{array}$ \\
\hline $\begin{array}{l}\text { Johannes C Van Der Wouden } \\
\text { Netherlands }\end{array}$ & $\begin{array}{l}\text { Harry Hao-Xiang Wang } \\
\text { UK }\end{array}$ & $\begin{array}{l}\text { Hakan Yaman } \\
\text { Turkey }\end{array}$ \\
\hline $\begin{array}{l}\text { Nikki Van Dessel } \\
\text { Netherlands }\end{array}$ & $\begin{array}{l}\text { Shengyu Wang } \\
\text { China }\end{array}$ & $\begin{array}{l}\text { John Yaphe } \\
\text { Portugal }\end{array}$ \\
\hline $\begin{array}{l}\text { Sonja Van Dillen } \\
\text { Netherlands }\end{array}$ & $\begin{array}{l}\text { Roger Webb } \\
\text { UK }\end{array}$ & $\begin{array}{l}\text { Ferdi Yavuz } \\
\text { Turkey }\end{array}$ \\
\hline $\begin{array}{l}\text { Sandra Van Dulmen } \\
\text { Netherlands }\end{array}$ & $\begin{array}{l}\text { Brandon Welch } \\
\text { USA }\end{array}$ & $\begin{array}{l}\text { Michael Yelland } \\
\text { Australia }\end{array}$ \\
\hline $\begin{array}{l}\text { Guido Van Hal } \\
\text { Belgium }\end{array}$ & $\begin{array}{l}\text { Matthias Wermeling } \\
\text { Germany }\end{array}$ & $\begin{array}{l}\text { Benjiamin Yip } \\
\text { Hong Kong }\end{array}$ \\
\hline $\begin{array}{l}\text { Oliver Van Hecke } \\
\text { UK }\end{array}$ & $\begin{array}{l}\text { Alison While } \\
\text { UK }\end{array}$ & $\begin{array}{l}\text { Zhaokang Yuan } \\
\text { China }\end{array}$ \\
\hline $\begin{array}{l}\text { Harm Van Marwijk } \\
\text { UK }\end{array}$ & $\begin{array}{l}\text { Anne Whittaker } \\
\text { UK }\end{array}$ & $\begin{array}{l}\text { Cristian Zeni } \\
\text { USA }\end{array}$ \\
\hline $\begin{array}{l}\text { Paul Van Royen } \\
\text { Belgium }\end{array}$ & $\begin{array}{l}\text { Sarah Wilke } \\
\text { Netherlands }\end{array}$ & $\begin{array}{l}\text { Yinsheng Zhang } \\
\text { China }\end{array}$ \\
\hline $\begin{array}{l}\text { Tjeerd Van Staa } \\
\text { UK }\end{array}$ & $\begin{array}{l}\text { Veronica Wilkie } \\
\text { UK }\end{array}$ & $\begin{array}{l}\text { Andrzej Zielinski } \\
\text { Sweden }\end{array}$ \\
\hline $\begin{array}{l}\text { Chris Van Weel } \\
\text { Australia }\end{array}$ & $\begin{array}{l}\text { Emily Williams } \\
\text { UK }\end{array}$ & $\begin{array}{l}\text { Nicholas Zwar } \\
\text { Australia }\end{array}$ \\
\hline $\begin{array}{l}\text { Isabelle Vedel } \\
\text { Canada }\end{array}$ & $\begin{array}{l}\text { Adam Windak } \\
\text { Poland }\end{array}$ & \\
\hline
\end{tabular}

